\title{
Face recognition is similarly affected by viewpoint in school- aged children and adults
}

\author{
Marisa Nordt ${ }^{\text {Corresp.., }}{ }^{1}$, Sarah Weigelt ${ }^{1}$ \\ ${ }^{1}$ Department of Developmental Neuropsychology, Institute of Psychology, Ruhr-Universität Bochum, Bochum, Germany \\ Corresponding Author: Marisa Nordt \\ Email address: marisa.nordt@rub.de
}

There is an ongoing debate on the question when face processing abilities mature. One aspect that has been part of this debate is the ability to recognize faces in and across different viewpoints. Here, we tested 128 participants consisting of school-age children (ages, 5 - 10 years) and adults (ages, 19-37 years) in two experiments to investigate the effects of different viewpoints (including front, three-quarter, profile view) on face recognition during development. Furthermore, we compared recognition performance for faces to that of another object category (cars). In the first experiment ( $n=88$ ) we tested if the pattern of performance for faces presented in different viewpoints is similar in schoolaged children and adults. Participants completed a two-alternative-forced-choice (2AFC) memory task comprising images of both faces and cars in front, three-quarter and profile view, which were presented in the same viewpoint during learning and testing. In the second experiment ( $n=40$ ) we tested if face recognition is similarly affected by viewpoint changes in children and adults. In this experiment the 2AFC memory task included a change of viewpoint between learning and testing. While in both experiments we found higher recognition performance for faces with increasing age, the overall pattern of both viewpoint and viewpoint-change-effects and also the difference between view-change- and no-change-conditions was similar across age groups. In contrast to faces, no viewpoint effects were observed in cars (experiment 1), viewpoint change effects, however, were similar for cars and faces (experiment 2 ). In sum, our results suggest early maturity of the ability to recognize faces in and across different viewpoints. 
1

2

3 Face recognition is similarly affected by viewpoint in school-aged children and adults

4

5

6

7

8

9

10

11

12 Correspondence concerning this article should be addressed to Marisa Nordt

13 email address: marisa.nordt@rub.de

14

15

16

17

18

19

20

21
Marisa Nordt and Sarah Weigelt

Department of Developmental Neuropsychology, Institute of Psychology, Ruhr-Universität Bochum, Universitätsstr. 150, 44801 Bochum, Germany

(1)

10

14

15

16




\section{Abstract}

25 There is an ongoing debate on the question when face processing abilities mature. One aspect that has been part of this debate is the ability to recognize faces in and across different

27 viewpoints. Here, we tested 128 participants consisting of school-age children (ages, $5-10$ years) and adults (ages, 19-37 years) in two experiments to investigate the effects of different viewpoints (including front, three-quarter, profile view) on face recognition during development. Furthermore, we compared recognition performance for faces to that of another object category (cars). In the first experiment $(n=88)$ we tested if the pattern of performance for faces presented in different viewpoints is similar in school-aged children and adults. Participants completed a two-alternative-forced-choice (2AFC) memory task comprising images of both faces and cars in

34 front, three-quarter and profile view, which were presented in the same viewpoint during 35 learning and testing. In the second experiment $(n=40)$ we tested if face recognition is similarly affected by viewpoint changes in children and adults. In this experiment the 2AFC memory task

37 included a change of viewpoint between learning and testing. While in both experiments we 38 found higher recognition performance for faces with increasing age, the overall pattern of both viewpoint and viewpoint-change-effects and also the difference between view-change- and nochange-conditions was similar across age groups. In contrast to faces, no viewpoint effects were observed in cars (experiment 1), viewpoint change effects, however, were similar for cars and

42 faces (experiment 2). In sum, our results suggest early maturity of the ability to recognize faces 43 in and across different viewpoints. 
Introduction

Even shortly after birth, newborns can distinguish their mothers face from the face of a stranger (Bushnell, Sai \& Mullin, 1989), thereby demonstrating that basic face recognition abilities are present at such an early point in life. Furthermore, it has been shown that during the first year of

52 life rapid changes with regard to face recognition abilities take place (Pascalis, de Haan \&

53 Nelson, 2002; Turati, Bulf \& Simion, 2008).

54 Nevertheless, there are also studies that support a prolonged development of face recognition 55 (Lawrence et al., 2008; Germine, Duchaine \& Nakayama, 2011; Weigelt et al., 2014). Studies 56 investigating unfamiliar face recognition from childhood to adulthood showed improvement of

57 face recognition abilities between six and 10 years, followed by a short plateau between the ages 58 of 10 and 13 (Carey, Diamond \& Woods, 1980; Lawrence et al., 2008). Germine, Duchaine and 59 Nakayama (2011) tested over 44000 participants aged 10 to 70 demonstrating that the ability to recognize faces increases even further, reaching its peak level around 31 years. In contrast,

61 performance levels for inverted faces peaked much earlier, at 23 years.

62 The question of early versus late maturity of face processing abilities has launched a debate in

63 face processing research. Early studies suggested that most, if not all core processes of face 64 recognition, like configural processing are not qualitatively present until age 10 years, thereby 65 promoting a late maturity view (Carey \& Diamond, 1977). However, when studies showed that 66 important processes of face recognition are present early, but performance does not reach adult 
67 levels until adolescence, the late maturity view was modified to a late quantitative maturity view

68 (Maurer, Grand \& Mondloch, 2002). As an alternative explanation, the early maturity hypothesis

69 has been proposed. It states that core aspects of face processing are present early, approximately

70 at the age of five years and, more importantly, are at adult levels at this point (Crookes \&

71 McKone, 2009).

72 This debate also encompasses the development of the ability to detect and recognize faces in and

73 across different viewpoints. This ability is of vital importance for our lives, as the viewpoint a

74 face is seen from changes from encounter to encounter with a person in everyday life. A main

75 finding from studies in adults on the effects of viewpoints is superior face recognition

76 performance for three-quarter views compared to front and profile views of faces (Bruce,

77 Valentine \& Baddeley, 1987; O’Toole, 1998; McKone, 2008). A further viewpoint effect is

78 lower recognition performance for profile views of faces, which is reflected in both longer

79 reaction times and lower accuracy (e.g., Bruce, Valentine \& Baddeley, 1987; McKone, 2008). It

80 has been suggested that this decrement of performance in comparison to front and three-quarter

81 views results from a lack of information on the configuration of internal features, like the spacing

82 of the eyes (Hill et al., 1997), and from a rarer occurrence of this view (McKone, 2008).

83 Several studies have addressed the development of face recognition across different viewpoints

84 during infancy. In a study by Turati, Bulf and Simion (2008), one- to three-day old infants were

85 habituated with a face in either front, three-quarter or profile view. In a next step, infants were

86 tested with two faces comprising the target face in a novel view (also either front, three-quarter

87 or profile) paired with a novel face presented in the same view. Turati and colleagues found a

88 significant novelty preference for faces despite viewpoint changes between front and three-

89 quarter view, however when profiles were included in either study or test phase no novelty 
90 preference was observed. This result is coherent with studies showing that the ability to encode

91 faces in profile view only emerges at seven months (Fagan III, 1976). Further support for this

92 finding comes from a near-infrared spectroscopy (NIRS) study in five- and eight-months-old

93 infants (Nakato et al., 2009). Results of this study showed that in eight-months-old infants the

94 concentration of oxy-hemoglobin $(\mathrm{oxy}-\mathrm{Hb})$ and of total $\mathrm{Hb}$ in right temporal brain regions

95 increased for front and profile views of faces (relative to a condition in which other objects were

96 shown), while for the younger age group concentration of oxy-Hb and total $\mathrm{Hb}$ increased for

97 front views of faces only. Thereby, these results suggest that eight-month-old but not five-

98 month-old infants showed face-like processing of profile views of faces in right temporal brain

99 regions. Together, behavioral as well as neuroimaging studies provide evidence that encoding of

100 profile views of faces is the only face processing ability undergoing qualitative changes during

101 infancy (for a review, see McKone, Crookes, Jeffery, \& Dilks, 2012).

102 Studies targeting the effects of viewpoint changes during childhood so far have focused on

103 changes between front and three-quarter view (Mondloch et al., 2003; Jeffery et al., 2013;

104 Anzures et al., 2014; Crookes \& Robbins, 2014). Mondloch et al. (2003) tested six-, eight-, and

105 10-year-old children and adults on five different tasks, in which participants had to match either

106 a certain identity despite changes in facial expression or changes in viewpoint, or they had to

107 match a certain direction of eye gaze, a facial expression or had to choose which faces were

108 mouthing certain vowels. While six-year-olds' performance was lower compared to adults on

109 each of these tasks, 10-year-olds' accuracy differed from that of adults only in one task:

110 matching of identities despite changes in viewpoint, thereby suggesting a late development of

111 this ability. In contrast, Crookes and Robbins (2014) found no indication for a developmental

112 change of face recognition across viewpoint change. They tested eight-year-old children and 
113 adults on a two-alternative forced-choice (2AFC) memory task including two conditions. In one

114 condition there was no change of viewpoint between study and test phase (same-view condition):

115 Faces were presented in front view in both study and test phase. In the second condition there

116 was a viewpoint change between study and test phase (change-view condition): Faces were

117 presented in front view in the study face and in three-quarter view in the test phase. Crookes and

118 Robbins found similar results for adults and eight-year-olds, with higher accuracy in the same-

119 view condition compared to the change-view condition in both groups, but showing neither a

120 main effect of age group nor an interaction between condition and age group, thus indicating

121 early maturity of face recognition across viewpoint changes. Likewise, Jeffery et al. (2013)

122 showed that the transfer of face aftereffects across changes in viewpoint was comparable in

123 seven- to nine-year-old children and adults. Face aftereffects refer to the altered perception of a

124 face after exposure to a preceding face. In the study by Jeffery et al. participants were adapted to

125 faces, which had been distorted by being contracted to the center of the face. In a next step

126 participants were tested with faces in either the same or different viewpoints. Adaptation to

127 distorted faces usually results in perceiving the test faces as distorted in the other direction, that

128 is to say, when the adapted face is contracted to the center, the test face will be perceived as

129 being distorted in the opposite way. Jeffery et al. found that children and adults showed similar

130 amounts of transfer across changes in face viewpoint.

131 The aim of the present study was to investigate viewpoint effects on face recognition during

132 childhood thereby adding to the previous literature in three respects. First, our study included

133 recognition of faces in profile views. As the ability to encode profile views of faces is the only

134 face processing ability undergoing qualitative changes during infancy (McKone, Crookes,

135 Jeffery, \& Dilks, 2012), this delayed development might still be visible during childhood. 
136 Second, in the present study we investigated viewpoint effects and viewpoint change effects

137 separately in two experiments. By viewpoint effects we refer to the effect a certain viewpoint has

138 on memory performance per se. These effects can be assessed when presenting a face in the

139 same viewpoint in study and test phase (experiment 1). Viewpoint change effects on the other

140 hand refer to the effects tested in paradigms, which include a change of the study phase to the

141 test phase (experiment 2). Separating viewpoint effects from viewpoint change effects, allows to

142 investigate if the pattern of performance for profile, three-quarter and front views as found in

143 adult studies, is the same in school-aged children. Moreover, it allows to disentangle if a

144 decrease in performance in a paradigm including a change of the viewpoint is driven by the

145 change of viewpoint or by effects of the viewpoint per se. Third, we compared the development

146 of viewpoint effects in face recognition to viewpoint effects for another object category (here:

147 cars) to assess the specificity of the viewpoint effects for faces.

148

149

150

151

152

153

154

155

156

157

158

\section{Experiment 1}

\section{Materials \& Methods}

\section{Participants}

120 healthy participants took part in this experiment. Data of four participants had to be excluded because of technical problems (two seven-year-olds and two nine-year-olds), because they were too old (one 11-year-old child) or because participants did not complete the experiment (one five-year-old and one six-year-old child). Data of further 25 children (13 five- to six-year-olds, nine seven- to eight-year-olds, three nine- to 10 -year-olds) were excluded because they kept looking away from the screen during the experiment or due to preemptive responses as indicated by reaction times shorter than $200 \mathrm{~ms}$ on some of their trials. Including these 25 participants, 
159 however, did not change the pattern of results. The remaining sample $(n=88)$ consisted of 19

160 five- and six-year-olds $(M=5.47 \mathrm{y}, S D=0.51,10$ male $), 25$ seven- and eight-year-olds $(M=7.52 \mathrm{y}$,

$161 S D=0.51,17$ male), 29 nine- and 10-year-olds $(M=9.48, S D=0.51,15$ male $)$ and 15 adults

$162(M=21.92 \mathrm{y}, S D=5.18 \mathrm{y}$, five male). Children were recruited via local primary schools and day-

163 care-centers and were rewarded with a little present after participation. Adult participants were

164 recruited by announcements at the university and received course credit as compensation. The

165 study was approved by the ethics committee of the faculty of Psychology of Ruhr-Universität

166 Bochum. Parents gave their written consent for their children's participation and adult

167 participants gave their written consent for their own participation.

168

169 Stimuli

170 Stimuli consisted of 180 photographs of faces and 180 photographs of cars. Images of faces were

171 taken from the Radboud Faces Database (Langner et al., 2010) and the Karolinska Directed

172 Emotional Faces database (Lundqvist, Flykt \& Öhman, 1998). The 180 photographs of faces

173 depict 60 Caucasian adults (30 female) with neutral facial expression shown from three

174 viewpoints: front view $\left(0^{\circ}\right)$, three-quarter view $\left(45^{\circ}\right)$ and profile view $\left(90^{\circ}\right)$. Photographs of cars

175 were taken from homepages of car manufacturers and include images of 60 different car models

176 in grey or silver shown in front, three-quarter and profile view. Images of faces and cars were

177 scaled to a maximum image height or width of 250 pixels and centered in a 300x300 pixels-sized

178 square with a grey background. Image processing was done using Adobe Photoshop CS5

179 Extended. Examples of the stimuli can be seen in Figure 1. 
180

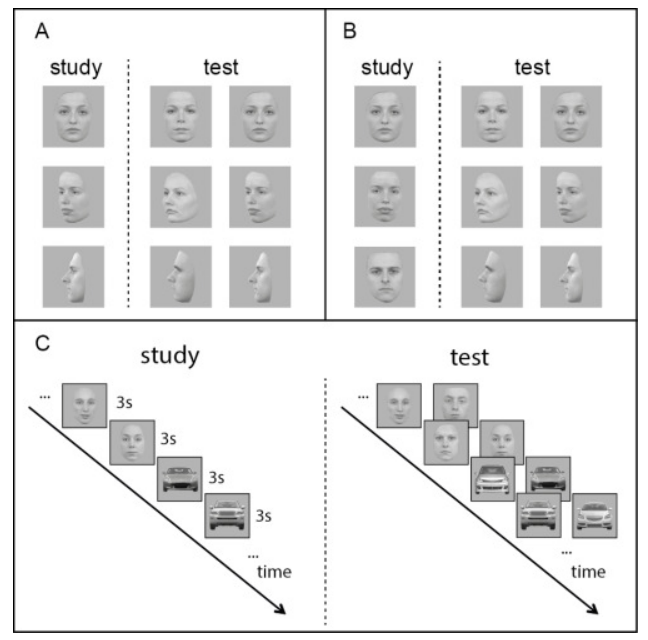

181 Figure 1. Stimuli and procedure. (A) Example face stimuli for the three task versions in experiment 1 (from top to bottom: front, three-quarter, profile). (B) Example face stimuli for the three task versions in experiment 2 (from top to bottom: $0^{\circ}$-, $45^{\circ}$-, $90^{\circ}$-change). (C) General overview on the task procedure.

\section{Design and Procedure}

187 The design of the memory task was adapted from Weigelt et al. (2014). Each participant took

188 part in three consecutive versions of this memory task; one version for each of the three

189 viewpoints (front, three-quarter, profile). Each of these tasks consisted of a study and a test phase

190 (see Figure 1c). In the study phase participants saw 10 images of one category (e.g., faces)

191 followed by 10 images of the other category (e.g., cars). Each image was presented for three

192 seconds and was immediately followed by the next image. The order of stimulus category was

193 counterbalanced between participants and was the same in the test phase, which consisted of a

194 2AFC task. The test phase was started by button press by the experimenter and was conducted 195 immediately after the study phase. Here, participants had to indicate the previously seen face or 196 car (target) via button press. Whenever an answer was given, the next pair of images appeared. 
197 Face targets were matched with distractor faces of the same sex. Also, no participant saw the

198 same face (or car) in more than one version of the task. We used three different stimulus sets to

199 rule out the influence of certain stimuli on the performance in certain conditions. The use of the

200 stimulus sets in the three conditions was counterbalanced between participants. Experiments

201 were conducted at the university, in primary schools, day-care-centers or at the families' houses.

202 Prior to the actual experiment children took part in a short practice test, using faces of cartoon

203 characters to make sure they had understood the procedure. If children did not answer the

204 practice trials correct, the practice task was repeated. Stimuli were presented on a MacBook Pro

205 with a screen size of 15" diagonally using MATLAB (version R2009b, The Mathworks) and the

206 Psychtoolbox (version 3.0.9, Brainard, 1997).

207

208 Analysis

209 Statistical analysis was done using SPSS (version 23). Unless stated otherwise the dependent 210 variable is accuracy in all analyses.

\section{Results}

\section{Preliminary analyses}

214 Controlling for the presentation order of stimulus categories

215 A repeated-measures ANOVA (rmANOVA) comprising the within-group-factors category

216 (faces, cars) and viewpoint (front, three-quarter, profile) and the between-group-factors age 
217 group (5-6, 7-8, 9-10, A) and category order (cars-faces, faces-cars) revealed no significant

218 effects of category order (all ps>.05). Therefore this factor was excluded from further analyses.

$219 \quad$ Testing for floor and ceiling effects

220 Floor and ceiling effects are common problems in developmental studies. Here, performance in

221 the youngest age group (five- and six-year-olds) differed significantly from chance (50\% correct)

222 in five out of six conditions. The only condition, which was not significantly different from

223 chance, was cars in three-quarter view, $t(18)=2.072, p=.053$. We interpret this finding as

224 evidence that also our youngest age group of children was in general capable of understanding

225 and performing our task. Adults' performance was significantly different from $100 \%$ in all

226 conditions (all ps<.05).

227 Main analyses

228

Effects of different viewpoints

229 We computed a rmANOVA comprising the within-group-factors category (faces, cars) and

230 viewpoint (front, three-quarter, profile) and the between-group-factor age group (5-6, 7-8, 9-10,

231 A). The rmANOVA revealed the following results: First, a significant main effect of viewpoint,

$232 F(2,168)=3.405, p=.036 ., \eta_{p}^{2}=.039$, demonstrating that memory performance was influenced

233 by the viewpoint stimuli were presented in. Second, a significant main effect of age group,

$234 F(3,84)=15.404, p<.001, \eta_{p}^{2}=.355$, reflecting higher memory performance with increasing

235 age of participants. Crucially, the interaction between viewpoint and age group was not

236 significant, $F(6,168)=0.922, p=.481$, thereby indicating similar viewpoint effects across age

237 groups (see Figure 2). Further significant effects were the main effect of category, $F(1,84)=$

$23823.984, p<.001, \eta_{p}^{2}=.222$, reflecting overall higher performance for faces compared to cars, and

239 the viewpoint $\mathrm{x}$ category interaction, $F(2,168)=4.084, p=.019, \eta_{p}^{2}=.046$, which showed that 
240 viewpoint effects differed for faces and cars. There were no other significant effects (category $\times$

241 age group, $F(3,84)=0.775, p=.511$; viewpoint $\times$ category $\times$ age group, $F(6,168)=1.019, p=$

$242.415)$

243 To further examine the viewpoint effect and the interaction between viewpoint and category, we 244 computed separate rmANOVAS (with the factors age group and viewpoint) on the face and car 245 data. In the car data, the effect of age group was significant, $F(3,84)=5.276, p=.002, \eta_{p}^{2}=.159$, 246 however, the effect of viewpoint was not significant, $F(2,168)=0.7, p=.498$, showing that the 247 viewpoint effect in the rmANOVA including both categories was mainly driven by the viewpoint 248 effect in faces. There were no other significant effects (viewpoint $\times$ age group, $F(6,168)=0.118$, $249 p=.994)$.

250 In the face data, there was also a significant effect of age group, $F(3,84)=18.578, p<.001, \eta_{p}^{2}$ $251=.399$, and a significant effect of viewpoint, $F(2,168)=6.41, p=.002, \eta_{p}^{2}=.071$, but no 252 significant viewpoint $\times$ age group interaction, $F(6,168)=1.721, p=.119, \eta_{p}^{2}=.058$. Post hoc t253 tests on the viewpoint effect in faces revealed that performance for three-quarter-views of faces 254 was significantly higher compared to performance for profile views, $t(87)=3.480, p=.001, d=$ 255 0.43. Likewise, performance for front views was significantly higher compared to profile views, $256 t(87)=3.185, p=.002, d=0.39$. Performance for front and three-quarter-views did not differ 257 significantly, $t(87)=-0.339, p=.736$. 
A

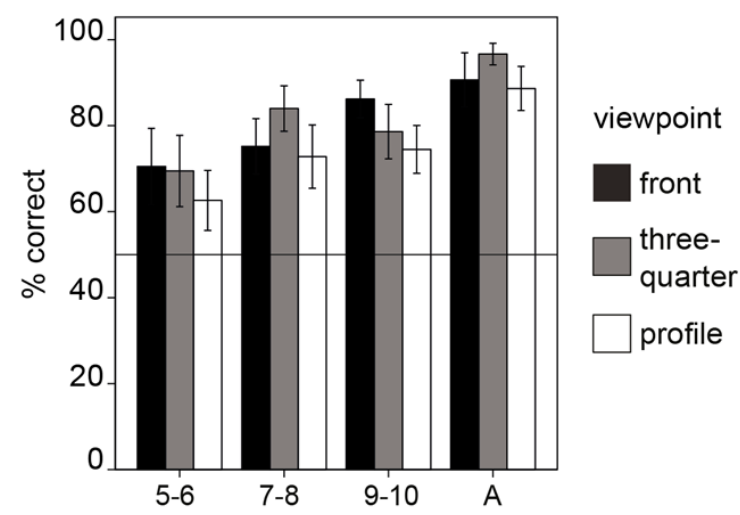

B

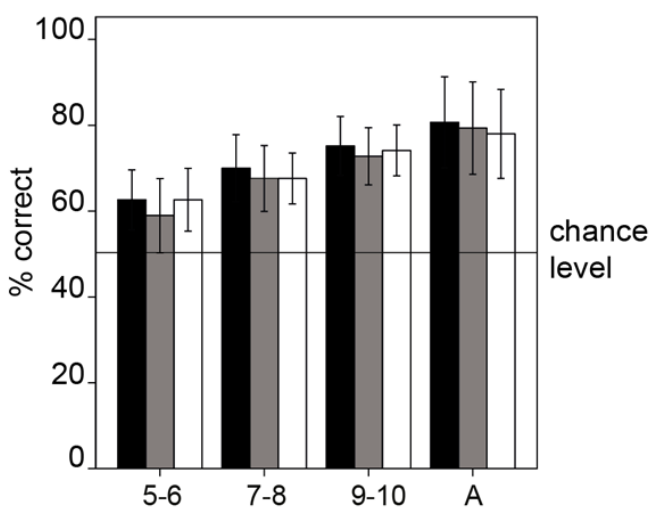

261 Figure 2. Results of experiment 1. Mean performance is shown for faces (A) and cars (B). The

262 age groups are indicated on the $\mathrm{x}$-axis and correspond to: five- to six-year-olds, seven- to eight-

263 year-olds, nine-to-10-year-olds and adults. Error bars: +/- 2 SE.

264

265

\section{Discussion}

266 Results from experiment 1 suggest that the pattern of face recognition performance for profile,

267 three-quarter and front views of faces is similar in school-age children and in adults. The lack of

268 a significant interaction between viewpoint and age group suggests that the pattern of

269 performance elicited by different viewpoints previously reported in studies in adults, i.e., lower

270 performance for profile view compared to both front and three-quarter views of faces (Bruce,

271 Valentine \& Baddeley, 1987), appears to be present in school-age children already.

\section{Experiment 2}

The goal of this experiment was to assess the influence of viewpoint changes on face memory performance and on memory performance for a control category (cars) in children and 276 adults.

\section{Materials \& Methods}




\section{Participants}

279 Participants consisted of a new group of participants $(n=40)$ that had not taken part in 280 experiment 1 . In this experiment we decided to test only the youngest age group comprising five-

281 to six-year-olds, because if we were to see developmental changes, we would expect them in the 282 youngest age group. Data of further 22 participants was excluded due to technical problems (one 283 adult, one child), because participants did not want to finish all three tasks (three children) and 284 participants were not able to perform the task (e.g., kept pressing only one button, did not look at 285 the screen or gave preemptive responses as indicated by reaction times shorter than $200 \mathrm{~ms}, 17$ 286 children). The remaining sample consisted of 18 five- to six-year-old children $(M=5.53 \mathrm{y}, S D=$ 2870.51 , five male) and 22 adults $(M=21.41 \mathrm{y}, S D=2.89$, two male). Recruitment and 288 compensation for the experiment was similar to experiment 1.

\section{Stimuli and Procedure}

290 Stimuli and procedure were identical to experiment 1.

\section{Design}

292 We used the same memory task as in experiment 1, however in this experiment we included two 293 conditions with a viewpoint change: In one condition, participants studied items in front view 294 and were tested in three-quarter-view, resulting in a $45^{\circ}$-change between study and test view 295 (45-condition). In the other condition, participants studied items in front view and were tested in 296 profile view, resulting in a $90^{\circ}$-change between study and test view ( $90^{\circ}$-condition).

297 Furthermore, participants completed one version of the memory task, in which —as in 298 experiment 1 - faces and cars were studied in front view and tested in front as well $\left(0^{\circ}\right.$ 299 condition), to allow for a direct comparison between conditions with and without viewpoint 
300 changes. The order of the three conditions $\left(0^{\circ}, 45^{\circ}, 90^{\circ}\right)$ was randomized between participants.

301 An overview of all three memory task versions can be seen in Figure 1b.

302 Results

303 Preliminary analyses

\section{Controlling for the presentation order of stimulus categories}

305 A rmANOVA including the within-subjects-factors category order (faces-cars, cars-faces), 306 category (faces, cars) and viewpoint-change $\left(0^{\circ}, 45^{\circ}, 90^{\circ}\right)$ and the between-subjects-factor age 307 group (children, adults) revealed no significant effects of category order (all $p>.05$ ). Therefore, 308 this factor was excluded from further analyses.

\section{Testing for floor and ceiling effects}

310 As in experiment 1 , children's performance differed significantly from chance in the $0^{\circ}$ -

311 viewpoint-change condition for both faces, $t(17)=4.096, p=.001, d=0.97$, and cars, $t(17)=$

$3122.406, p=.028, d=0.57$. Furthermore, the $45^{\circ}$-condition was significantly different from chance 313 for faces, $t(17)=3.173, p=.006, d=0.75$, and marginally significant for cars, $t(17)=2.012, p=$ $314.060, d=0.47$. The $90^{\circ}$-condition was at chance for both faces, $t(17)=0.483, p=.636$, and cars

$315 t(17)=-.559, p=.584$. We conclude that, although children's performance was at chance in some 316 conditions, in general children were able to perform the task. Adult data was free from floor or 317 ceiling effects in all conditions (all $p \mathrm{~s}<.05$ ).

\section{Comparison of results from experiment 1 and 2}

319 As the $0^{\circ}$-condition in experiment 2 is equivalent to the front-condition in experiment 1 , we 320 tested in a first step, if results in five- to six-year-olds and adults was similar across experiments.

321 There were no differences in either adults or five- to six-year-olds in performance for front views 
322 of faces or front views of cars from experimen1 1 to experiment 2 (all $p s>.05$ ), showing that

323 performance was similar for the groups in experiment 1 and 2.

324

325 Main analyses

326 Effects of viewpoint changes

327 We computed a rmANOVA including the within-group-factors category (faces, cars), viewpoint-

328 change $\left(0^{\circ}, 45^{\circ}, 90^{\circ}\right)$ and the between-group-factor age group (children, adults), which revealed

329 the following findings: First, a main effect of viewpoint change, $F(2,76)=20.328, p<.001, \eta_{p}^{2}$

$330=.349$, showing that memory performance for faces and cars was influenced by changes in

331 viewpoint. Second, a main effect of age group, $F(1,38)=36.032, p<.001, \eta_{p}^{2}=.487$, indicating

332 overall better performance for adults than children. Importantly, the interaction between age

333 group and viewpoint change was not significant, $F(2,76)=0.81, p=.922$, indicating that

334 memory performance was similarly affected by viewpoint changes in five- to six-year-old

335 children and adults (see Figure 3). Furthermore, results revealed a main effect of category,

$336 F(1,38)=17.327, p<.001, \eta_{p}^{2}=.313$, indicating overall higher performance for faces compared to

337 cars. No other interactions were significant $\left(\right.$ category $\times$ age group, $F(1,38)=1.297, p=.262, \eta_{p}^{2}$

$338=.033 ;$ category $\times$ viewpoint change, $F(2,76)=1.267, p=.287$; category $\times$ viewpoint change $\times$

339 age group, $F(2,76)=.091, p=.913)$.

340 To further examine the nature of the viewpoint change effect, we computed post hoc t-tests to

341 compare the three conditions $\left(0^{\circ}, 45^{\circ}, 90^{\circ}\right)$. Memory performance in the $0^{\circ}$-condition differed

342 significantly from the $90^{\circ}$-condition, $t(39)=6.413, p<.001, d=1.13$, and performance in the

$34345^{\circ}$-condition differed significantly from the $90^{\circ}$-condition, $t(39)=5.013, p<.001, d=0.54$. 
344 The difference in performance between the $0^{\circ}$-condition and the $45^{\circ}$-condition was not

345 significant, $t(39)=1.691, p=.099$.

346

A

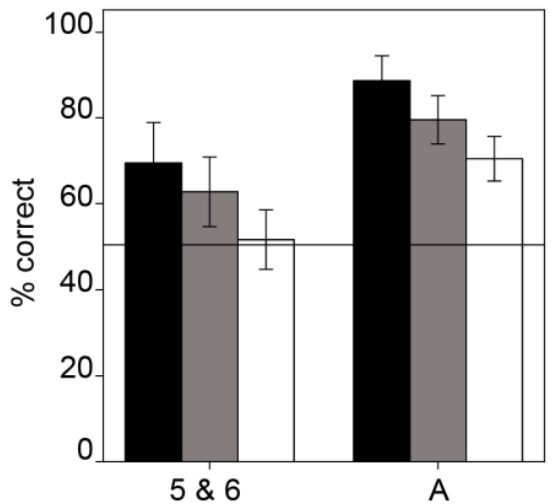

B

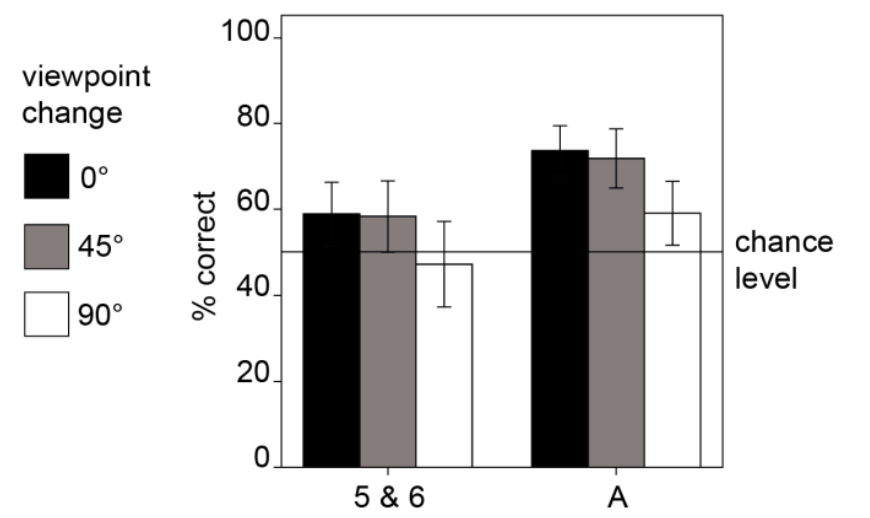

Figure 3. Results of experiment 2 for faces (A) and cars (B). The age groups are indicated on the $\mathrm{x}$-axis and correspond to: five-and six-year-olds and adults. Error bars: +/- 2 SE.

\section{Discussion}

352 Results from experiment 2 show that the effects of viewpoint changes are similar in five- to six353 year-old children and adults. These findings are in line with previous results showing that eight-

354 year-old children and adults were similarly affected by a rotation between front and three-quarter 355 views of faces (Crookes \& Robbins, 2014). Furthermore, our results extend these findings by 356 showing that also a viewpoint change involving profile views similarly affects face recognition 357 five- to six-year-old children and adults. A limitation to the present findings is that five- and sixyear-old children's performance was at chance for the $90^{\circ}$-condition, which might have masked a stronger decline in performance for this group compared to the adult group. Moreover, the exclusion rate in this experiment was higher compared to experiment 1 . The reason for this high exclusion rate might be found in the higher difficulty in task 2 compared to task 1 which is also 
362 reflected in the performance drop in adults for the conditions involving a viewpoint change. For

363 instance, adults have an accuracy of $89 \%$ in the most difficult face-condition in experiment 1

364 (faces in profile view), but performance drops to about $70 \%$ for the $90^{\circ}$-change condition in

365 experiment 2 . This difficulty might have influenced children's motivation to properly complete

366 all three tasks. Nevertheless, comparable levels of performance in the $0^{\circ}$-change condition in

367 experiment 2 with the same condition in experiment 1 (front-condition), suggest that the data

368 was not negatively influenced by the exclusion rate.

369

370

371

\section{General Discussion}

372 The present study investigated the effects of different viewpoints and viewpoint changes on face

373 recognition performance in school-age-children and adults. The main finding of this study is that

374 face recognition in children and adults is similarly affected by viewpoint effects (experiment 1)

375 and also by viewpoint change effects (experiment 2). Thereby, the present findings are consistent

376 with recent findings suggesting no developmental differences of view-invariant face recognition

377 (Jeffery et al., 2013; Crookes \& Robbins, 2014). Our results extend previous findings by

378 showing that not only the general difference between encoding in the same and across views, but

379 also the pattern of how recognition performance is affected by different viewpoints (experiment

380 1) and viewpoint changes (experiment 2) appears to be similar between school-age children and

381 adults. Furthermore, our results show that also when including profile views of faces, viewpoint

382 effects and viewpoint change effects are similar in children and adults.

383 In experiment 1 we assessed the pattern of recognition performance for each of the three

384 viewpoints front, three-quarter and profile. Besides an age-related increase in performance, we 
385 found that recognition of faces, but not of cars was influenced by the viewpoint the stimulus was

386 presented in. For the face data, performance in five- to 10-year-old children and adults

387 performance for both front and three-quarter views was significantly higher compared to that for

388 profile views, a pattern that has been found in numerous studies in adults (e.g., Bruce et al.,

389 1987; McKone, 2008). Also with regard to developmental aspects, profile views can be assigned

390 a special role, as the ability to encode faces in profile view - in contrast to encoding of front and

391 three-quarter views - only emerges at seven months (Fagan III, 1976) and therefore seems to be

392 one of few abilities undergoing qualitative changes. However, the present results suggest that

393 after that qualitative change has taken place, the pattern of performance for profile, three-quarter

394 and front views seems to be quite stable at least from child- to adulthood.

395 In contrast to faces, for cars, we did not observe viewpoint effects. One factor that seems to be

396 relevant for performance differences between several viewpoints of objects is the familiarity with

397 certain viewpoints (see, Blanz et al., 1999). So a possible explanation for the absence of

398 viewpoint effects in cars in the present study would be that in normal life we see cars roughly

399 equally often from all three tested views.

400 In experiment 2 we investigated the effects of viewpoint changes on recognition performance.

401 We found an overall increase of performance with age, a significant effect of viewpoint change

402 but no interaction between viewpoint change and age, showing that viewpoint change effects

403 were similar across the age groups. In the face data, all three conditions differed significantly

404 from each other with the performance being highest for the $0^{\circ}$-condition, lower for the $45^{\circ}$ -

405 condition and with lowest performance for the $90^{\circ}$-condition. This finding matches results from

406 previous studies in adults reporting that when participants learned front views of faces,

407 performance decreased with increasing angle between study and test phase (Hill, Schyns \& 
408 Akamatsu, 1997). Also, results from experiment 2 show that the decrease of performance in a 409 condition containing a viewpoint change $\left(45^{\circ}\right.$ - and $90^{\circ}$ - condition $)$ compared to a condition

410 without change $\left(0^{\circ}\right.$-condition $)$ is similar in children and adults, indicating that five-and six-year-

411 old children's representations of faces are not more view-specific than those of adults. Thereby

412 our results are consistent with a study by Crookes and Robbins (2014), who found that eight-

413 year-old children and adults were similarly affected by a viewpoint change (study front and test

414 three-quarter) compared to a condition without viewpoint change (study front and test front). The

415 present findings conflict with results from studies suggesting that the ability to recognize faces

416 across different views matures late (Mondloch et al., 2003). A possible explanation for the

417 divergence of results is that the study by Mondloch et al. (2003) also included up and down

418 rotations of the face and not only changes between front and three-quarter views.

419 A limitation to the present study is that overall performance was lower for cars compared to

420 faces, indicating that the car stimuli were more difficult, probably due to the selection of images

421 of very similar car models. Ideally, overall performance levels for faces and cars would have

422 been matched. Furthermore, in experiment 2 children were at chance for both faces and cars in

423 the $90^{\circ}$-condition, showing that this condition was too difficult for this age group. However,

424 since performance was not at chance in the $45^{\circ}$-condition we can conclude that in general also

425 the youngest age group was able to perform the task and that the low performance in the $90^{\circ}$ -

426 condition is indeed informative.

427

428 Conclusion

429 In conclusion, the present study shows similar viewpoint and viewpoint-change-effects on face-

430 memory-performance in school-age children and adults. Our results suggest that the pattern of 
431 performance for profile, three-quarter and front views of faces seems to be quite stable at least

432 from child- to adulthood and that face recognition is similarly affected by changes across these

433 views in children and adults. In sum, our results suggest early maturity of the ability to recognize

434 faces in and across different viewpoints.

435

436

437 Acknowledgements

438 We thank Astrid Hönekopp, Tobias Meißner, Luzie Mount, Helen Prüfer, Rebecka Röhnke,

439 Katharina Sommer, Sophia Terwiel and Ricarda F. Weiland for their assistance in data

440 collection.

441

442

443

444

445

446

447

448

449

450

451

452

453 
454

455

456

457

458

459

460

461

462

\section{References}

463

Anzures G., Kelly DJ., Pascalis O., Quinn PC., Slater AM., de Viviés X., Lee K. 2014. Own- and

464 other-race face identity recognition in children: The effects of pose and feature

465 composition. Developmental Psychology 50:469-481. DOI: 10.1037/a0033166.

466

467

Blanz V., Tarr MJ., Bülthoff HH., Vetter T. 1999. What object attributes determine canonical views? Perception-London 28:575-600.

468 Brainard DH. 1997. The psychophysics toolbox. Spatial vision 10:433-436.

469 Bruce V., Valentine T., Baddeley AM. 1987. The basis of the 3/4 view advantage in face 470 recognition. Applied cognitive psychology 1:109-120.

471 Bushnell IWR., Sai F., Mullin JT. 1989. Neonatal recognition of the mother's face. British 472 Journal of Developmental Psychology 7:3-15. DOI: 10.1111/j.2044-

473 835X.1989.tb00784.x.

474 Carey S., Diamond R. 1977. From piecemeal to configurational aspects of face processing. $475 \quad$ Science 195:312-314. 
476 Carey S., Diamond R., Woods B. 1980. Development of face recognition: A maturational

477 component? Developmental Psychology 16:257-269. DOI: 10.1037/0012-1649.16.4.257.

478 Crookes K., McKone E. 2009. Early maturity of face recognition: No childhood development of 479 holistic processing, novel face encoding, or face-space. Cognition 111:219-247. DOI:

480 10.1016/j.cognition.2009.02.004.

481

482

483

484

485

486

487

488

489

490

491

492

493

494

495

496

497

498

recognition: Evidence from 8-year-olds and adults. Journal of Experimental Child Psychology 126:103-111. DOI: 10.1016/j.jecp.2014.03.010.

Fagan III JF. 1976. Infants' recognition of invariant features of faces. Child Development:627638.

Germine LT., Duchaine B., Nakayama K. 2011. Where cognitive development and aging meet: Face learning ability peaks after age 30. Cognition 118:201-210. DOI: 10.1016/j.cognition.2010.11.002.

Hill H., Schyns PG., Akamatsu S. 1997. Information and viewpoint dependence in face recognition. Cognition 62:201-222.

Jeffery L., Rathbone C., Read A., Rhodes G. 2013. Children's face identity representations are no more view specific than those of adults. Journal of Experimental Psychology: Human Perception and Performance 39:450-463. DOI: 10.1037/a0029141.

Langner O., Dotsch R., Bijlstra G., Wigboldus DHJ., Hawk ST., van Knippenberg A. 2010. Presentation and validation of the Radboud Faces Database. Cognition \& Emotion 24:1377-1388. DOI: 10.1080/02699930903485076.

Lawrence K., Bernstein D., Pearson R., Mandy W., Campbell R., Skuse D. 2008. Changing abilities in recognition of unfamiliar face photographs through childhood and 
499

500

501

502

503

504

505

506

507

508

509

510

511

512

513

514

515

516

517

518

519

520

adolescence: Performance on a test of non-verbal immediate memory (Warrington RMF) from 6 to 16 years. Journal of Neuropsychology 2:27-45. DOI:

$10.1348 / 174866407 X 231074$.

Lundqvist D., Flykt A., Öhman A. 1998. The Karolinska Directed Emotional Faces KDEF. Stockholm, Sweden: Department of Clinical Neuroscience, Psychology section, Karolinska Institutet.

Maurer D., Grand RL., Mondloch CJ. 2002. The many faces of configural processing. Trends in cognitive sciences 6:255-260.

McKone E. 2008. Configural processing and face viewpoint. Journal of Experimental Psychology: Human Perception and Performance 34:310-327. DOI: 10.1037/00961523.34.2.310.

McKone E., Crookes K., Jeffery L., Dilks DD. 2012. A critical review of the development of face recognition: Experience is less important than previously believed. Cognitive Neuropsychology 29:174-212. DOI: 10.1080/02643294.2012.660138.

Mondloch CJ., Geldart S., Maurer D., Grand RL. 2003. Developmental changes in face processing skills. Journal of Experimental Child Psychology 86:67-84. DOI: $10.1016 / \mathrm{S} 0022-0965(03) 00102-4$.

Nakato E., Otsuka Y., Kanazawa S., Yamaguchi MK., Watanabe S., Kakigi R. 2009. When do infants differentiate profile face from frontal face? A near-infrared spectroscopic study. Human Brain Mapping 30:462-472. DOI: 10.1002/hbm.20516.

O’Toole AJ. 1998. Stimulus-specific effects in face recognition over changes in viewpoint. Vision Research 38:2351-2363. 
521 Pascalis O., de Haan M., Nelson CA. 2002. Is Face Processing Species-Specific During the First $522 \quad$ Year of Life? Science 296:1321-1323. DOI: 10.1126/science.1070223.

523 Turati C., Bulf H., Simion F. 2008. Newborns' face recognition over changes in viewpoint. 524 Cognition 106:1300-1321. DOI: 10.1016/j.cognition.2007.06.005.

525 Weigelt S., Koldewyn K., Dilks DD., Balas B., McKone E., Kanwisher N. 2014. Domain526 specific development of face memory but not face perception. Developmental Science 527 17:47-58. DOI: 10.1111/desc.12089.

528

529 